\title{
A Blind Adaptive Decorrelating Detector for CDMA Systems
}

\author{
Sennur Ulukus, Student Member, IEEE, and Roy D. Yates, Member, IEEE
}

\begin{abstract}
The decorrelating detector is known to eliminate multiaccess interference when the signature sequences of the users are linearly independent, at the cost of enhancing the Gaussian receiver noise. In this paper, we present a blind adaptive decorrelating detector which is based on the observation of readily available statistics. The algorithm recursively updates the filter coefficients of a desired user by using the output of the current filter. Due to the randomness of the information bits transmitted and the ambient Gaussian channel noise, the filter coefficients evolve stochastically. We prove the convergence of the filter coefficients to a decorrelating detector in the mean squared error (MSE) sense. We develop lower and upper bounds on the MSE of the receiver filter from the convergence point and show that with a fixed step size sequence, the MSE can be made arbitrarily small by choosing a small enough step size. With a time-varying step size sequence, the MSE converges to zero implying an exact convergence. The proposed algorithm is distributed, in the sense that no information about the interfering users such as their signature sequences or power levels is needed. The algorithm requires the knowledge of only two parameters for the construction of the receiver filter of a desired user: the desired user's signature sequence and the variance of the additive white Gaussian (AWG) receiver noise. This detector, for an asynchronous code division multiple access (CDMA) channel, converges to the one-shot decorrelating detector.
\end{abstract}

Index Terms-Code division multiple access (CDMA), decorrelating detector, multiuser detection.

\section{INTRODUCTION}

C ODE division multiple access (CDMA) systems suffer from the near-far effect because of the nonorthogonality of the users' signature sequences. Multiuser detection [1] can be used to overcome the near-far problem by exploiting the known structure of the multiple access interference to effectively demodulate the nonorthogonal signals of the users. It was shown, in [2], that the optimal multiuser detector has a computational complexity which increases exponentially with the number of active users. Several suboptimum detectors have been proposed to achieve a performance comparable to that of the optimum detector, while keeping the complexity

Manuscript received September 1, 1997; revised February 1, 1998. This work was supported by the NSF under Grant NCRI 95-06505. This work was presented in part at the IEEE Global Telecommunications Conference, Phoenix, AZ, November 1997.

S. Ulukus was with the Department of Electrical and Computer Engineering, Wireless Information Network Laboratory (WINLAB), Rutgers University, Piscataway, NJ. She is now with the Wireless Research Department, AT\&T Laboratories, Red Bank, NJ 07701 USA.

R. D. Yates is with the Department of Electrical and Computer Engineering, Wireless Information Network Laboratory (WINLAB), Rutgers University, Piscataway NJ 08855-0909 USA.

Publisher Item Identifier S 0733-8716(98)07900-1. low. Examples include: the decorrelating detector [3]; the decision feedback detector [4]; the minimum mean squared error (MMSE) detector [5]; and the multistage detectors [6].

The decorrelating detector [3], [7] achieves the same asymptotic efficiency as the optimal multiuser detector and has linear (in terms of the number of users) computational complexity. The decorrelating detector was shown to eliminate the multiaccess interference totally, if the signature sequences of the users are linearly independent, at the cost of enhancing the additive white Gaussian (AWG) receiver noise. The decorrelating detector of [3] and [7] is centralized and noniterative. The construction of the decorrelating detector filter for a certain user requires the knowledge of the signature sequences of all the interfering users, as well as the signature sequence of the user of interest. In addition, for an $N$-user system, the construction requires inversion of the $N \times N$ crosscorrelation matrix. Blind adaptive algorithms are desirable in order to overcome the need for knowledge about the parameters of the interfering users, and iterative algorithms are needed to avoid the matrix inversion which may have a large computational complexity. A blind adaptive algorithm based on the minimization of the output energy was given in [8]. This algorithm was shown to converge to the MMSE multiuser detector [5].

In [9], an adaptive multiuser detector which converges to the decorrelating detector is proposed. This detector still needs the signature sequences of all users. In [10], blind algorithms based on signal subspace tracking are investigated and two algorithms which converge to the decorrelating and MMSE multiuser detectors are proposed. The blind adaptive decorrelating detector (BADD) proposed in [10] needs information about the variance of the AWG channel noise and the number of users, both of which can be estimated, again, by using the subspace tracking techniques. The computational complexity of the algorithm of [10] is $O(G N)$ per iteration where $G$ is the processing gain and $N$ is the number of active users.

In this paper, we present a blind adaptive multiuser detector which uses observables that are readily available at the receiver and which converges to a decorrelating detector. The detector is constructed via a distributed iterative algorithm, which updates the receiver filter coefficients of a desired user by using the previous output of the filter under construction. Since the filter output is random, due to the randomness of the multiaccess interference and existence of ambient Gaussian channel noise, the algorithm evolves stochastically. We prove the convergence of the filter coefficients to a decorrelating detector in the mean squared error (MSE) sense. We develop lower and upper bounds for the MSE of the filter coefficients 
at iteration $n+1$, in terms of the MSE of the filter coefficients at iteration $n$ and investigate the conditions under which the MSE sequence converges to zero. Two kinds of algorithms, using a fixed step size sequence and a time dependent step size sequence, are proposed. For the algorithm using a fixed step size, we obtain the lower and upper bounds for the limiting MSE as $n$ approaches infinity in terms of the step size. We show that the limiting MSE can be made arbitrarily small by choosing a small enough step size. On the other hand, we directly prove the convergence of limiting MSE to zero when a time-varying step size sequence of a special structure is used.

The computational complexity of the proposed algorithm is $O(G)$ per iteration. The proposed algorithm requires the knowledge of only two parameters for the construction of the filter coefficients for a desired user: the desired user's signature sequence and the variance of the AWG noise (AWGN). The variance of the AWGN is a fixed quantity (not time varying) and can be estimated easily, perhaps before the information transfer starts, when only the samples of AWGN can be observed without any interference at the output of an arbitrary (nonzero) receiver filter. In such a case, a reliable estimate of the variance of the AWGN can be obtained by timeaveraging the squares of the output of the receiver filter. Another implementation alternative is to allocate a unit energy filter that is orthogonal to all the of the signatures being used. In this case, again, the square of the output of the filter will be an unbiased estimate for the noise variance. In the latter case, since the filter is orthogonal to all the signatures, one can continue to have estimates for the noise variance while the users are transmitting and the decorrelating receiver filter coefficients are being updated. Until Section VI we will assume that the noise variance is known perfectly. In Section VI we investigate the effects of using estimated values of noise variance on the convergence of the proposed algorithm.

In the following section we will briefly summarize the well known decorrelating detector and develop the necessary background for the presentation of the BADD.

\section{The Decorrelating Detector}

Until Section V, where the convergence of the proposed algorithm for an asynchronous CDMA channel will be investigated, we will assume a synchronous CDMA system. Throughout this paper binary phase-shift keying (BPSK) modulation is assumed in order to simplify the analysis. We will use $G$ dimensional vector $\boldsymbol{s}_{i}$ to denote the preassigned unique signature sequence of user $i$. Let us define an $N \times G$ dimensional matrix $S$ with its $(i, j)$ th element being $\left(\boldsymbol{s}_{i}\right)_{j}$, the $j$ th component of $\boldsymbol{s}_{i}$. Therefore, the rows of $S$ (equivalently the columns of $S^{\top}$ ) are the signature sequences of the users. For future use, we define a subspace $\mathcal{L}$ in $G$ dimensional vector space to be the subspace spanned by the signature sequences of the users, i.e.,

$$
\mathcal{L}=\operatorname{span}\left\{\boldsymbol{s}_{1}, \cdots, \boldsymbol{s}_{N}\right\}=\text { column space of } \boldsymbol{S}^{\top} .
$$

We consider the decorrelating detector for the first user, without loss of generality, and represent it by a $G$ dimensional vector $c$. Then $\boldsymbol{c}$ should satisfy the following condition:

$$
\boldsymbol{S c}=\alpha \boldsymbol{e}_{1}
$$

where $e_{1}$ is the first unit vector in $N$ dimensional space, i.e., $\boldsymbol{e}_{1}=\left[\begin{array}{lllll}1 & 0 & 0 & \cdots & 0\end{array}\right]^{\top}$ and $\alpha$ is a nonnegative real number. Equation (2) states that the receiver filter for the desired (first) user should be orthogonal to the signature sequences of all the interfering users, and it should have a nonzero correlation with the desired user. Here, this nonzero correlation is equal to the scaling factor $\alpha$. It is not difficult to show that the bit error rate (BER) performance of the decorrelating detector is insensitive to scaling of the filter, as long as the filter eliminates the multiaccess interference totally. The reason for this is that the scalar factor multiplies both the received power level of the desired user and the AWGN. Thus, for any nonnegative value of $\alpha$, we have a decorrelating detector with the same BER performance. The importance of using $\alpha \neq 1$ will be evident in the next section, where we will avoid the need for the knowledge about the received power of the desired user by a particular selection of $\alpha$.

We first note that (2) has more than one solution, because it has $N$ equalities and $G$ unknowns and, typically, $G \gg N$. The unique decorrelating detector for the first user, $\boldsymbol{d}$, is given in [3] as

$$
\boldsymbol{d}=\boldsymbol{S}^{\top}\left(\boldsymbol{S} \boldsymbol{S}^{\top}\right)^{-1} \boldsymbol{e}_{1}
$$

Let us denote any solution of (2) as $\overline{\boldsymbol{c}}$ then, by inserting (2) into (3), we obtain (assuming $\alpha=1$ for the time being)

$$
\begin{aligned}
\boldsymbol{d} & =\boldsymbol{S}^{\top}\left(S \boldsymbol{S}^{\top}\right)^{-1} \boldsymbol{S c} \\
& =\boldsymbol{P} \overline{\boldsymbol{c}} .
\end{aligned}
$$

Note that $P=S^{\top}\left(S S^{\top}\right)^{-1} S$ is the projection matrix which projects any vector onto the column space of $S^{\top}$. Also note that the column space of $S^{\top}$ is the subspace spanned by $\boldsymbol{s}_{1}, \cdots, \boldsymbol{s}_{N}$, i.e., the subspace which was previously denoted as $\mathcal{L}$. Therefore, although (2) has more than one solution, all of the solutions have the same projection onto $\mathcal{L}$ and this projection is equal to the unique decorrelating detector solution of [3].

Let $\Lambda$ be an $N \times N$ dimensional diagonal matrix, with the received power of user $i, p_{i}$ being its $i$ th diagonal element. Multiplying both sides of (2), first with $\boldsymbol{\Lambda}$ and then with $\boldsymbol{S}^{\top}$ we obtain

$$
\boldsymbol{S}^{\top} \boldsymbol{A S c}=\alpha p_{1} \boldsymbol{s}_{1}
$$

We observe that although (6) has $G$ equations in $G$ unknowns, it does not have a unique solution for $c$, since $(G-N)$ eigenvalues of $S^{\top} A S$ are equal to zero. The solution spaces of (2) and (6) are related as stated in the following lemma.

Lemma 1: If $\boldsymbol{s}_{1}, \cdots, \boldsymbol{s}_{N}$ are linearly independent, then all solutions of (2) and (6) coincide.

A proof for Lemma 1 is given in the Appendix.

Let us define $A=\boldsymbol{S}^{\top} \boldsymbol{A} \boldsymbol{S}$. At this point we choose $\alpha=1 / p_{1}$ and devise the following gradient descent algorithm:

$$
c(n+1)=c(n)-\mu\left(A c(n)-s_{1}\right)
$$


Note that the iterative algorithm given in (7) converges to the solution of (6) and equivalently, by Lemma 1, to the solution of (2). The fact that the solution of (2) is not unique is noted earlier. Note that, for any $\boldsymbol{c}$

$$
P A c=S^{\top}\left(S S^{\top}\right)^{-1} S S^{\top} A S c=S^{\top} \Lambda S c=A c .
$$

This means that for any $c(n), A c(n)$ lies entirely in $\mathcal{L}$. Since $\boldsymbol{s}_{1} \in \mathcal{L}$ by definition, the correction term added to $\boldsymbol{c}(n)$ at every iteration in (7) is always in $\mathcal{L}$. Starting from $n=0$, by induction we obtain

$$
c(n)=\boldsymbol{P c}(n)+\boldsymbol{c}(0)-P \boldsymbol{c}(0) .
$$

Therefore, if the iterative algorithm (7) is started in the subspace $\mathcal{L}$, implying $\boldsymbol{c}(0)=\boldsymbol{P c}(0)$, then from (9) we will have $\boldsymbol{c}(n)=\boldsymbol{P} \boldsymbol{c}(n)$ for all $n$. In this case, $\boldsymbol{c}(n)$ will always stay in $\mathcal{L}$, and it will converge to the scaled version of the unique decorrelating detector solution $\boldsymbol{c}^{*}=\left(1 / p_{1}\right) \boldsymbol{d}$ as $n$ goes to infinity. Note that the algorithm converges to the scaled version of $\boldsymbol{d}$, the decorrelating detector of [3] and [7], instead of converging to $\boldsymbol{d}$ because $\alpha$ was chosen to be $1 / p_{1}$ instead of 1 . Note also that $\boldsymbol{d}$ and $\boldsymbol{c}^{*}=\left(1 / p_{1}\right) \boldsymbol{d}$ have the same BER performance as discussed earlier.

The restriction that the iterations should be started in $\mathcal{L}$, in order for algorithm (7) to converge to a decorrelating detector, is fairly mild. Selections $\boldsymbol{c}(0)=\mathbf{0}, \boldsymbol{c}(0)=\boldsymbol{s}_{1}$ or any linear combination of the signature sequences imply $c(0) \in \mathcal{L}$, satisfying the convergence condition of (7). The signature sequences of all of the users must be known for the algorithm given in (7). Also, the algorithm of (7) is an off-line algorithm in the sense that it does not utilize any real time measurements or observations. The algorithm of (7) can be run before the real information transmission of mobiles to the base station start. After running the algorithm for some time, the filter coefficients would converge to the decorrelating detector filter and then the communication can be started. In this paper, our aim is to develop a blind adaptive algorithm which would converge to the decorrelating detector solution, in a stochastic sense, by using real random measurements while the users are active and transmitting bits. To this end, we will propose an algorithm which can be viewed as the stochastic version of the deterministic algorithm given in (7).

\section{A BADD}

The received base band signal before the receiver filters can be written as

$$
\boldsymbol{r}=\sum_{i=1}^{N} \sqrt{p_{i}} a_{i} \boldsymbol{s}_{i}+\boldsymbol{n}
$$

where $a_{i}$ is the information bit ( \pm 1 , equiprobably), $p_{i}$ is the power, $\boldsymbol{s}_{i}$ is the signature sequence of user $i$, and $\boldsymbol{n}$ is a Gaussian random vector with zero mean and $E\left[\boldsymbol{n} \boldsymbol{n}^{\top}\right]=\sigma^{2} \boldsymbol{I}$. Note that

$$
E\left[\boldsymbol{r} \boldsymbol{r}^{\top}\right]=\sum_{i=1}^{N} p_{i} \boldsymbol{s}_{i} \boldsymbol{s}_{i}^{\top}+\sigma^{2} \boldsymbol{I}=\boldsymbol{A}+\sigma^{2} \boldsymbol{I}
$$

Therefore, using (11), the deterministic iteration of (7) can be written in an exact form as

$$
\boldsymbol{c}(n+1)=\boldsymbol{c}(n)-\mu\left[\left(E\left[\boldsymbol{r} \boldsymbol{r}^{\top}\right]-\sigma^{2} \boldsymbol{I}\right) \boldsymbol{c}(n)-\boldsymbol{s}_{1}\right] .
$$

Note that $\boldsymbol{r} \boldsymbol{r}^{\top}-\sigma^{2} \boldsymbol{I}$ is an unbiased estimate for $A$ matrix. In order to obtain a practical algorithm, we replace $E\left[\boldsymbol{r} \boldsymbol{r}^{\top}\right]$ in (12) with the estimate $\boldsymbol{r}(n) \boldsymbol{r}^{\top}(n)$ where $\boldsymbol{r}(n)$ is the received signal vector at the $n$th iteration

$$
\boldsymbol{c}(n+1)=\boldsymbol{c}(n)-\mu\left[\left(\boldsymbol{r}(n) \boldsymbol{r}^{\top}(n)-\sigma^{2} \boldsymbol{I}\right) \boldsymbol{c}(n)-\boldsymbol{s}_{1}\right] .
$$

Before analyzing the convergence of (13), we state it in terms of available observations and list the parameters needed at each iteration. We note that the output of the receiver filter of the desired user at time $n$ is $y(n)=\boldsymbol{r}^{\top}(n) \boldsymbol{c}(n)$. Thus, the implementation oriented version of the algorithm (13) is

$$
\boldsymbol{c}(n+1)=\left(1+\sigma^{2} \mu\right) \boldsymbol{c}(n)-\mu\left(\boldsymbol{r}(n) y(n)-\boldsymbol{s}_{1}\right) .
$$

Since $\boldsymbol{r}(n)$ and $y(n)$ are readily available at the input and output of the receiver filter that is under construction, only two system parameters are needed to be known in order to run the algorithm: the signature sequence of the desired user $\boldsymbol{s}_{1}$ and the variance of the AWGN $\sigma^{2}$. The variance of the AWGN is a fixed quantity which can be easily estimated before the communication starts by using the outputs of a nonzero receiver filter as discussed in Section I.

\section{CONVERGENCE OF THE BADD}

In this section we will investigate the convergence of the BADD proposed in Section III. Let us define the zero mean random vector $\eta(n)$ as

$$
\eta(n)=\left(\boldsymbol{r} \boldsymbol{r}^{\top}-\boldsymbol{A}-\sigma^{2} \boldsymbol{I}\right) \boldsymbol{c}(n) .
$$

Noting that $A \boldsymbol{c}^{*}=\boldsymbol{s}_{1}$, we can write the stochastic iterations (13) and (14) as

$$
\boldsymbol{c}(n+1)=\boldsymbol{c}(n)-\mu\left[\boldsymbol{A}\left(\boldsymbol{c}(n)-\boldsymbol{c}^{*}\right)+\boldsymbol{\eta}(n)\right] .
$$

Subtracting $\boldsymbol{c}^{*}$ from both sides of (16) and defining $\epsilon(n)=$ $c(n)-c^{*}$ we obtain

$$
\boldsymbol{\epsilon}(n+1)=\boldsymbol{\epsilon}(n)-\mu(\boldsymbol{A} \boldsymbol{\epsilon}(n)+\boldsymbol{\eta}(n)) .
$$

Note that $\|\epsilon(n)\|^{2}$, the norm of $\epsilon(n)$, is a measure of the distance of the receiver filter at iteration $n$ from the convergence point. In order to relate the distance measures at iterations $n$ and $n+1$, we square both sides of (17) and obtain

$$
\begin{aligned}
\|\boldsymbol{\epsilon}(n+1)\|^{2}= & \|\boldsymbol{\epsilon}(n)\|^{2}-2 \mu \boldsymbol{\epsilon}(n)^{\top} \boldsymbol{A} \boldsymbol{\epsilon}(n) \\
& +2 \mu^{2} \boldsymbol{\epsilon}(n)^{\top} \boldsymbol{A} \boldsymbol{\eta}(n)-2 \mu \boldsymbol{\epsilon}(n)^{\top} \boldsymbol{\eta}(n) \\
& +\mu^{2} \boldsymbol{\epsilon}(n)^{\top} \boldsymbol{A}^{2} \boldsymbol{\epsilon}(n)+\mu^{2}\|\boldsymbol{\eta}(n)\|^{2} .
\end{aligned}
$$

Taking the conditional expectation of both sides of (18), conditioned on $\boldsymbol{\epsilon}(n)=\boldsymbol{\epsilon}$, and observing that $E[\boldsymbol{\eta}(n) \mid \boldsymbol{\epsilon}(n)=$ $\boldsymbol{\epsilon}]=\mathbf{0}$ yields

$$
\begin{aligned}
E\left[\|\epsilon(n+1)\|^{2} \mid \epsilon(n)=\right. & \boldsymbol{\epsilon}]=\|\boldsymbol{\epsilon}\|^{2}-2 \mu \epsilon^{\top} A \boldsymbol{\epsilon}+\mu^{2} \epsilon^{\top} A^{2} \epsilon \\
& +\mu^{2} E\left[\|\eta(n)\|^{2} \mid \boldsymbol{\epsilon}(n)=\boldsymbol{\epsilon}\right] .
\end{aligned}
$$

We will be using the results of the following lemmas to develop bounds for the right-hand side of (19). 
Lemma 2: If $\boldsymbol{P} \boldsymbol{\epsilon}=\mathbf{0}$ then $\boldsymbol{\epsilon}^{\top} \boldsymbol{A \epsilon}=0$ and $\boldsymbol{\epsilon}^{\top} \boldsymbol{A}^{2} \boldsymbol{\epsilon}=0$. If $\boldsymbol{P} \epsilon \neq \mathbf{0}$ then there exist $0<k_{0} \leq k_{1}<\infty$, such that

$$
\begin{aligned}
& k_{0}\|\boldsymbol{\epsilon}\|^{2} \leq \boldsymbol{\epsilon}^{\top} \boldsymbol{A} \boldsymbol{\epsilon} \leq k_{1}\|\boldsymbol{\epsilon}\|^{2} \text { and } \\
& k_{0}^{2}\|\boldsymbol{\epsilon}\|^{2} \leq \boldsymbol{\epsilon}^{\top} \boldsymbol{A}^{2} \boldsymbol{\epsilon} \leq k_{1}^{2}\|\boldsymbol{\epsilon}\|^{2} .
\end{aligned}
$$

Lemma 3: There exist $0<c_{0} \leq c_{1}<\infty$, such that

$$
0 \leq E\left[\|\boldsymbol{\eta}(n)\|^{2} \mid \boldsymbol{\epsilon}(n)=\boldsymbol{\epsilon}\right] \leq c_{0}+c_{1}\|\epsilon\|^{2} .
$$

The proofs of Lemmas 2 and 3 can be found in the Appendix.

If $P \boldsymbol{\epsilon}=\mathbf{0}$, we can develop the following lower and upper bounds for the right-hand side of (19), using Lemmas 2 and 3

$$
\begin{aligned}
& E\left[\|\boldsymbol{\epsilon}(n+1)\|^{2} \mid \boldsymbol{\epsilon}(n)=\boldsymbol{\epsilon}, \boldsymbol{P} \boldsymbol{\epsilon}=\mathbf{0}\right] \leq\left(1+\mu^{2} c_{1}\right)\|\boldsymbol{\epsilon}\|^{2}+c_{0} \mu^{2} \\
& E\left[\|\epsilon(n+1)\|^{2} \mid \boldsymbol{\epsilon}(n)=\boldsymbol{\epsilon}, \boldsymbol{P} \boldsymbol{\epsilon}=\mathbf{0}\right] \geq\|\boldsymbol{\epsilon}\|^{2} .
\end{aligned}
$$

If $\boldsymbol{P} \boldsymbol{\epsilon} \neq \mathbf{0}$, the following lower and upper bounds for the right-hand side of (19) can be obtained, using, again, Lemmas 2 and 3

$$
\begin{aligned}
& E\left[\|\boldsymbol{\epsilon}(n+1)\|^{2} \mid \boldsymbol{\epsilon}(n)=\boldsymbol{\epsilon}, \boldsymbol{P} \boldsymbol{\epsilon} \neq \mathbf{0}\right] \leq\left(1-2 \mu k_{0}+\left(k_{1}^{2}+c_{1}\right) \mu^{2}\right) \\
& \text { - }\|\epsilon\|^{2}+c_{0} \mu^{2} \\
& E\left[\|\boldsymbol{\epsilon}(n+1)\|^{2} \mid \boldsymbol{\epsilon}(n)=\boldsymbol{\epsilon}, \boldsymbol{P} \boldsymbol{\epsilon} \neq \mathbf{0}\right] \geq\left(1-2 \mu k_{1}+k_{0}^{2} \mu^{2}\right)\|\boldsymbol{\epsilon}\|^{2} .
\end{aligned}
$$

An important observation toward the convergence proof is that the projection of $\boldsymbol{c}(n)$ (equivalently the projection of $\boldsymbol{\epsilon}(n)$ ) on $\mathcal{L}$ would be nonzero almost surely (a.s.) [11]. This means that for any $n$, the probability of the event $[\boldsymbol{P} \epsilon(n)=\mathbf{0}]$ is zero. Similar to the deterministic result in (9), it can be shown, using induction on (17), that (see also [12, eq. (16)])

$$
\boldsymbol{\epsilon}(n)=\boldsymbol{P} \boldsymbol{\epsilon}(n)+\boldsymbol{\epsilon}(0)-\boldsymbol{P} \boldsymbol{\epsilon}(0) \text { a.s. }
$$

If $\epsilon(0) \in \mathcal{L}$ then $\epsilon(0)=P \epsilon(0)$ and (26) can be written as

$$
\boldsymbol{\epsilon}(n)=\boldsymbol{P} \boldsymbol{\epsilon}(n) \text { a.s. }
$$

Note that $\boldsymbol{\epsilon}(0)=\boldsymbol{c}(0)-\boldsymbol{c}^{*}$, since $\boldsymbol{c}^{*} \in \mathcal{L}, \boldsymbol{\epsilon}(0) \in \mathcal{L}$ is equivalent to $\boldsymbol{c}(0) \in \mathcal{L}$. Thus, (27) is valid if iteration (13) is started in $\mathcal{L}$. The upper bound on $E\left[\|\boldsymbol{\epsilon}(n+1)\|^{2} \mid \boldsymbol{\epsilon}(n)=\boldsymbol{\epsilon}\right]$ can be developed as follows:

$$
\begin{aligned}
E\left[\|\boldsymbol{\epsilon}(n+1)\|^{2} \mid \boldsymbol{\epsilon}(n)=\boldsymbol{\epsilon}\right] & \\
= & E\left[\|\boldsymbol{\epsilon}(n+1)\|^{2} \mid \boldsymbol{\epsilon}(n)=\boldsymbol{\epsilon}, \boldsymbol{P \epsilon}=\mathbf{0}\right] \operatorname{Prob}[P \boldsymbol{\epsilon}=\mathbf{0}] \\
& +E\left[\|\boldsymbol{\epsilon}(n+1)\|^{2} \mid \boldsymbol{\epsilon}(n)=\boldsymbol{\epsilon}, \boldsymbol{P \epsilon} \neq \mathbf{0}\right] \operatorname{Prob}[\boldsymbol{P \epsilon} \neq \mathbf{0}] \\
= & E\left[\|\boldsymbol{\epsilon}(n+1)\|^{2} \mid \boldsymbol{\epsilon}(n)=\boldsymbol{\epsilon}, \boldsymbol{P} \boldsymbol{\epsilon} \neq \mathbf{0}\right] \\
\leq & \left(1-2 \mu k_{0}+\left(k_{1}^{2}+c_{1}\right) \mu^{2}\right)\|\boldsymbol{\epsilon}\|^{2}+c_{0} \mu^{2}
\end{aligned}
$$

where we used the facts $\operatorname{Prob}[\boldsymbol{P \epsilon}=\mathbf{0}]=0$ and $\operatorname{Prob}[\boldsymbol{P} \boldsymbol{F} \neq$ $\mathbf{0}]=1$ and the result in (24). Following similar steps to those in (28), and using the result given in (25), this time we can obtain the following lower bound for the same term:

$$
E\left[\|\epsilon(n+1)\|^{2} \mid \epsilon(n)=\epsilon\right] \geq\left(1-2 \mu k_{1}+k_{0}^{2} \mu^{2}\right)\|\epsilon\|^{2} .
$$

Taking the expectation of both sides of the inequalities in (28) and (29), with respect to $\boldsymbol{\epsilon}(n)$, and letting $b_{n}=E\left[\|\boldsymbol{\epsilon}(n)\|^{2}\right]$, we obtain the following lower and upper bounds for $b_{n+1}$, the mean squared error (MSE) of the filter coefficients at iteration $n+1$ from $\boldsymbol{c}^{*}$, in terms of $b_{n}$

$$
\begin{aligned}
& \left(1-2 \mu k_{1}+k_{0}^{2} \mu^{2}\right) b_{n} \\
& \quad \leq b_{n+1} \leq\left(1-2 \mu k_{0}+\left(k_{1}^{2}+c_{1}\right) \mu^{2}\right) b_{n}+c_{0} \mu^{2} .
\end{aligned}
$$

By defining

$$
\alpha_{0}=1-2 \mu k_{0}+\left(k_{1}^{2}+c_{1}\right) \mu^{2} \quad \alpha_{1}=1-2 \mu k_{1}+k_{0}^{2} \mu^{2}
$$

we can rewrite (30) as

$$
\alpha_{1} b_{n} \leq b_{n+1} \leq \alpha_{0} b_{n}+c_{0} \mu^{2} .
$$

We observe from (32) that the nonnegative sequence $b_{n}$ is sandwiched between the two sequences generated according to $b_{n+1}^{\prime}=\alpha_{0} b_{n}^{\prime}+c_{0} \mu^{2}$ and $b_{n+1}^{\prime \prime}=\alpha_{1} b_{n}^{\prime \prime}$. These two sequences converge to finite numbers if and only if $\mu$ is chosen such that $\left|\alpha_{0}\right|<1$ and $\left|\alpha_{1}\right|<1$. Note that both $\alpha_{0}$ and $\alpha_{1}$ are equal to 1 at $\mu=0$. We also note that both $\alpha_{0}$ and $\alpha_{1}$ are locally decreasing as $\mu$ increases, since

$$
\left.\frac{d \alpha_{0}}{d \mu}\right|_{\mu=0}=-2 k_{0}<0 \quad \text { and }\left.\quad \frac{d \alpha_{1}}{d \mu}\right|_{\mu=0}=-2 k_{1}<0 .
$$

This means that we can always choose $\mu$ small enough so that

$$
\left|\alpha_{0}\right|<1 \text { and }\left|\alpha_{1}\right|<1
$$

in which case the sequences $b_{n}^{\prime}$ and $b_{n}^{\prime \prime}$ converge and the limiting MSE, i.e., $\lim _{n \rightarrow \infty} b_{n}$, has finite lower and upper bounds. From the sandwich theorem, we have

$$
0 \leq \lim _{n \rightarrow \infty} b_{n} \leq \frac{c_{0} \mu^{2}}{1-\alpha_{0}} .
$$

We can evaluate the value of the upper bound in the extreme case, when $\mu \rightarrow 0$, as

$$
\lim _{\mu \rightarrow 0} \frac{c_{0} \mu^{2}}{1-\alpha_{0}}=\lim _{\mu \rightarrow 0} \frac{c_{0} \mu}{2 k_{0}-\left(k_{1}^{2}+c_{1}\right) \mu}=0 .
$$

Therefore, if the step size $(\mu)$ is chosen to be extremely small, then the MSE of the filter coefficients from the convergence point goes to zero as the number of iterations grows to infinity. But note that as $\mu \rightarrow 0$, the numbers $\alpha_{0}$ and $\alpha_{1}$ go to one, in which case the convergence rate goes to zero. Thus, we observe the tradeoff between the limiting value of the MSE and the convergence rate. If a large value is chosen as the step size $\mu$, then the convergence rate is faster, but the limiting MSE is larger and, if a small value is chosen as the step size, the limiting MSE is smaller, but the convergence rate is slower as well. Hence, a time-dependent step size sequence, which takes large values at the beginning and smaller values at the end, may be preferable. An iteration index $(n)$-dependent step size sequence can be used to accomplish this. Replacing the fixed step size $\mu$ in (14) with the time varying step size sequence $a_{n}$, we obtain the new algorithm

$$
\boldsymbol{c}(n+1)=\left(1+\sigma^{2} a_{n}\right) \boldsymbol{c}(n)-a_{n}\left(\boldsymbol{r}(n) y(n)-\boldsymbol{s}_{1}\right) .
$$

If the step size sequence satisfies two simple conditions, known as Robbins-Monro conditions [13] as shown in (38), then 


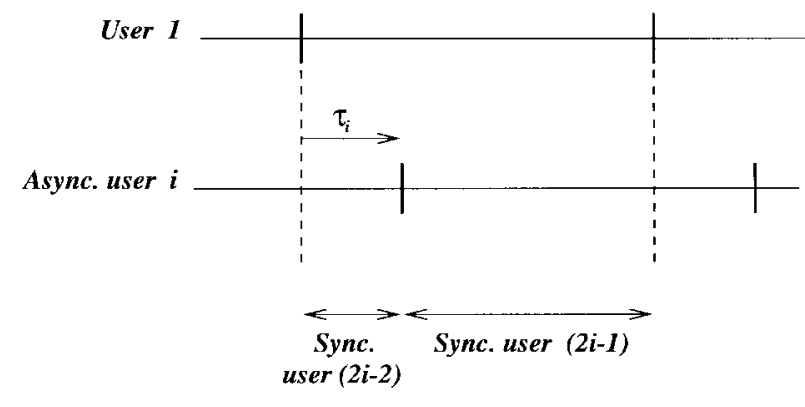

Fig. 1. Asynchronous CDMA model.

the filter coefficients converge to the decorrelating detector solution in the MSE sense

$$
\sum_{n}^{\infty} a_{n}=\infty \text { and } \sum_{n}^{\infty} a_{n}^{2}<\infty
$$

The convergence is stated in the following lemma, which is proved in the Appendix.

Lemma 4: Stochastic iteration given in (37) converges to $c^{*}$ in the MSE sense, i.e.,

$$
\lim _{n \rightarrow \infty} E\left[\left\|\boldsymbol{c}(n)-c^{*}\right\|^{2}\right]=0
$$

if $a_{n}$ satisfies the conditions of (38).

Note that $a_{n}=a /\left(n+n_{0}\right)$, for any $a>0$ and $n_{0}>0$ is a sequence which satisfies (38).

\section{THE BADD IN AN AsYNCHRONOUS CDMA SYSTEM}

Let $\tau_{i}$ denote the delay of the $i$ th user. Without loss of generality, we will assume that $\tau_{1}$, the desired user's delay, is equal to zero and $\tau_{i} \geq 0$, for $i>1$. As in [1], each asynchronous interfering user $i$ can be replaced with two synchronous users $2 i-2$ and $2 i-1$, with modified signature sequences (see Fig. 1). The modified signature sequences, $\overline{\boldsymbol{s}}_{\boldsymbol{i}}$, of these synchronous users are given as

$$
\begin{aligned}
& \bar{s}_{2 i-2}= \begin{cases}s_{i}(t) & 0 \leq t \leq \tau_{i} \\
0 & \tau_{i}<t \leq T_{b}\end{cases} \\
& \bar{s}_{2 i-1}= \begin{cases}0 & 0 \leq t \leq \tau_{i} \\
s_{i}(t) & \tau_{i}<t \leq T_{b}\end{cases}
\end{aligned}
$$

where $T_{b}$ is the bit duration. In this case the chip sampled received signal before the receiver filter of the desired user at the $n$th bit interval is given as

$$
\boldsymbol{r}(n)=\sum_{i=1}^{N} \sqrt{p_{i}}\left(a_{i}(n-1) \overline{\boldsymbol{s}}_{2 i-2}+a_{i}(n) \bar{s}_{2 i-1}\right)+n
$$

where $a_{i}(n)$ denotes the information bit transmitted by user $i$ in the $n$th bit interval. In (39), for convenience, the desired (first) user is also written in terms of the signature of two equivalent users. Note that the signature sequence $\bar{s}_{0}$ is identically zero and $\bar{s}_{1}=s_{1}$.
From the mutual independence of the transmitted bits and the AWGN we have

$$
\begin{aligned}
E\left[\boldsymbol{r}(n) \boldsymbol{r}^{\top}(n)\right] & =\sum_{i=1}^{N} p_{i}\left(\overline{\boldsymbol{s}}_{2 i-2} \overline{\boldsymbol{s}}_{2 i-2}^{\top}+\overline{\boldsymbol{s}}_{2 i-1} \overline{\boldsymbol{s}}_{2 i-1}^{\top}\right)+\sigma^{2} \boldsymbol{I} \\
& =\sum_{i=1}^{2 N-1} \bar{p}_{i} \overline{\boldsymbol{s}}_{i} \overline{\boldsymbol{s}}_{i}^{\top}+\sigma^{2} \boldsymbol{I} \\
& =\bar{A}+\sigma^{2} \boldsymbol{I}
\end{aligned}
$$

where $\bar{p}_{2 i-2}$ and $\bar{p}_{2 i-1}$ are equal to $p_{i}$, since they denote the received power levels of the two synchronous users representing the asynchronous user $i$.

If the stochastic iteration (13) is used for the asynchronous system described in this section, the receiver filter of the desired user will converge to the point $\bar{c}^{*}$ where

$$
\bar{A} \bar{c}^{*}=s_{1} .
$$

We define a $(2 N-1) \times G$ matrix $\bar{S}$, with its $i$ th row being $\overline{\boldsymbol{s}}_{i}$. Thus, $\overline{\boldsymbol{S}}$ contains the signature sequences of the equivalent synchronous system with $(2 N-1)$ users. Equation (41) is equivalent to

$$
\bar{S}^{\top} \bar{\Lambda} \bar{S} \bar{c}^{*}=s_{1}
$$

where $\bar{\Lambda}$ is a $(2 N-1) \times(2 N-1)$ dimensional diagonal matrix with $\overline{\boldsymbol{\Lambda}}_{i i}=\bar{p}_{i}$. By Lemma 1 the solution of (42) is equal to the solution of

$$
\overline{\boldsymbol{S}} \overline{\boldsymbol{c}}^{*}=\boldsymbol{e}_{1}
$$

if the rows of $\bar{S}$, equivalently the modified signature sequences, are linearly independent. This condition is the linear independence assumption (LIA) of [7], which requires ( $2 N-$ 1) $\leq G$. In this paper, for the asynchronous channel, we will assume that the LIA is in effect, as was done in [7].

The convergence point $\bar{c}^{*}$ is the one-shot decorrelator [2], [7] which is the decorrelating receiver filter when only one bit of the desired user is considered. The one-shot decorrelator has lower complexity than asynchronous decorrelating detectors, which take into account the dependence of the received signal in different bit intervals. The one-shot decorrelating detector, however, does suffer some loss in performance.

\section{EFFECTS OF NOISE VARIANCE ESTIMATION}

The proposed algorithm (14) requires that the variance of the AWGN is known and used in the update of the filter coefficients. In this section, we investigate the effect of missing the exact value of noise variance $\sigma^{2}$.

In wireless communication channels, unlike other parameters, such as channel gains that are subject to fading, noise variance is a fixed parameter. Although it is fixed, it is unknown and needs to be estimated. There can be two approaches.

1) The noise variance can be estimated before the information transmission hence the update of the filter coefficients starts and this estimate (a fixed deterministic number) is used in every update of the filter. 
2) A real time estimator which produces a new (random) estimate for the noise variance can be built, and its estimates can be used in the filter updates. The physical implementation of this estimator can be realized in many ways. One method could be to allocate a unit energy filter $\boldsymbol{v}$ which is orthogonal to all signature sequences being used by the users and use the output of this filter for estimating $\sigma^{2}$. Since the filter is orthogonal to all signature sequences, the square of its output is equal to

$$
z=\left(\boldsymbol{v}^{\top} \boldsymbol{n}\right)^{2}
$$

where $\boldsymbol{n}$ is the same as the $\boldsymbol{n}$ in (10). Note that, since $\boldsymbol{v}$ has unit energy, $E[z]=\sigma^{2}$ and $z$ is an unbiased estimate of $\sigma^{2}$.

Whether an estimate of $\sigma^{2}$ is obtained before the bit transmission starts and this same fixed estimate is used in every update of the filter coefficients, or a new unbiased estimate is used at each filter update is an implementation issue. Below, we will investigate the performance of the proposed algorithm in both cases.

\section{A. Estimating $\sigma^{2}$ Before Filter Updates}

In this section we assume that a deterministic number $\tilde{\sigma}^{2}$ is used in place of $\sigma^{2}$ in (14) [equivalently, in (13)]. From (13), the update equation becomes

$$
\boldsymbol{c}(n+1)=\boldsymbol{c}(n)-\mu\left[\left(\boldsymbol{r}(n) \boldsymbol{r}^{\top}(n)-\tilde{\sigma}^{2} \boldsymbol{I}\right) \boldsymbol{c}(n)-s_{1}\right] .
$$

The deterministic version of the algorithm becomes

$$
\boldsymbol{c}(n+1)=\boldsymbol{c}(n)-\mu\left[\tilde{A} \boldsymbol{c}(n)-\boldsymbol{s}_{1}\right]
$$

where

$$
\tilde{\boldsymbol{A}}=\boldsymbol{S}^{\top} \boldsymbol{\Lambda} \boldsymbol{S}+\left(\sigma^{2}-\tilde{\sigma}^{2}\right) \boldsymbol{I}=\sum_{i=1}^{N} p_{i} \boldsymbol{s}_{i} \boldsymbol{s}_{i}^{\top}+\left(\sigma^{2}-\tilde{\sigma}^{2}\right) \boldsymbol{I} .
$$

If $\tilde{\sigma}^{2} \leq \sigma^{2}$, then $\tilde{A}$ is a positive semidefinite matrix and the algorithm in (46) converges deterministically and the one in (45) in the MSE sense to $\tilde{e}$ satisfying

$$
\tilde{A} \tilde{c}=s_{1} .
$$

If $\tilde{\sigma}^{2}<\sigma^{2}$, then $\tilde{A}$ is invertible and

$$
\tilde{\boldsymbol{c}}=\tilde{A}^{-1} \boldsymbol{s}_{1} .
$$

The convergence of the stochastic iteration (45) to $\tilde{\boldsymbol{c}}$ can be shown by writing (45) as

$\boldsymbol{c}(n+1)=\boldsymbol{c}(n)-\mu\left[\left(\boldsymbol{r}(n) \boldsymbol{r}^{\top}(n)-\tilde{A}+\tilde{A}-\tilde{\sigma}^{2} \boldsymbol{I}\right) \boldsymbol{c}(n)-\boldsymbol{s}_{1}\right]$

so that (48) implies

$$
\begin{gathered}
\boldsymbol{c}(n+1)=\boldsymbol{c}(n)-\mu\left[\tilde{A}(\boldsymbol{c}(n)-\tilde{\boldsymbol{c}})+\left(\boldsymbol{r}(n) \boldsymbol{r}^{\top}(n)\right.\right. \\
\left.\left.-\left(\tilde{\boldsymbol{A}}+\tilde{\sigma}^{2} \boldsymbol{I}\right)\right) \boldsymbol{c}(n)\right] .
\end{gathered}
$$

Note that the random term $\left(\boldsymbol{r}(n) \boldsymbol{r}^{\top}(n)-\left(\tilde{\boldsymbol{A}}+\tilde{\sigma}^{2} \boldsymbol{I}\right)\right) \boldsymbol{c}(n)$ in (51) is identical to $\eta(n)$ defined in (15) and, thus, (51) can be written as

$$
\boldsymbol{c}(n+1)=\boldsymbol{c}(n)-\mu[\tilde{A}(\boldsymbol{c}(n)-\tilde{\boldsymbol{c}})+\boldsymbol{\eta}(n)] .
$$

Subtracting $\tilde{\boldsymbol{c}}$ from both sides of (52) and defining $\tilde{\boldsymbol{\epsilon}}(n)$ as $\tilde{\boldsymbol{\epsilon}}(n)=\boldsymbol{c}(n)-\tilde{\boldsymbol{c}}$ yields

$$
\tilde{\boldsymbol{\epsilon}}(n+1)=\tilde{\boldsymbol{\epsilon}}(n)-\mu(\tilde{A} \tilde{\boldsymbol{\epsilon}}(n)+\boldsymbol{\eta}(n)) .
$$

Comparing (53) with (17), we observe that Lemmas 2 and 3 are still valid and that the algorithm converges in the MSE sense. Note, as well, that since there is a unique solution for $\tilde{\boldsymbol{c}}$ when $\tilde{\sigma}^{2} \neq \sigma^{2}$, the arguments involving the projection onto signal space are not necessary. Also not necessary is the condition that the algorithm should be started in the signal space.

Note that $\tilde{\boldsymbol{c}}$ in (49) is a scaled version of the MMSE multiuser detector, where a reduced value for the noise variance is used, i.e., $\left(\sigma^{2}-\tilde{\sigma}^{2}\right)$ is used instead of the true noise variance $\sigma^{2}$.

In two extreme cases the detector $\tilde{\boldsymbol{c}}$ reduces to a well known multiuser detector: if $\tilde{\sigma}^{2}=0$ then $\tilde{\boldsymbol{c}}$ is the true MMSE multiuser detector and if $\tilde{\sigma}^{2}=\sigma^{2}$ then $\tilde{\boldsymbol{c}}$ is the decorrelating multiuser detector. Defining $\tilde{A}_{1}$ as $\tilde{\boldsymbol{A}}_{1}=\sum_{i \neq 1}^{N} p_{i} \boldsymbol{s}_{i} \boldsymbol{s}_{i}^{\top}+\left(\sigma^{2}-\right.$ $\left.\tilde{\sigma}^{2}\right) \boldsymbol{I}$, the filter in (49) can be written as $\tilde{\boldsymbol{c}}=\beta \tilde{\boldsymbol{A}}_{1}^{-1} \boldsymbol{s}_{1}$, where $\beta$ is a positive factor. The signal to interference ratio (SIR) of the desired (first) user is given by

$$
\begin{aligned}
\mathrm{SIR}_{1} & =\frac{p_{1}\left(\tilde{\boldsymbol{c}}^{\top} \boldsymbol{s}_{1}\right)^{2}}{\sum_{j \neq 1} p_{j}\left(\tilde{\boldsymbol{c}}^{\top} \boldsymbol{s}_{j}\right)^{2}+\sigma^{2}\left(\tilde{\boldsymbol{c}}^{\top} \tilde{\boldsymbol{c}}\right)} \\
& =\frac{p_{1} \tilde{\boldsymbol{c}}^{\top} \boldsymbol{s}_{1} \boldsymbol{s}_{1}^{\top} \tilde{\boldsymbol{c}}}{\tilde{\boldsymbol{c}}^{\top}\left(\sum_{j \neq 1} p_{j} \boldsymbol{s}_{j} \boldsymbol{s}_{j}^{\top}+\sigma^{2} \boldsymbol{I}\right) \tilde{\boldsymbol{c}}} \\
& =\frac{p_{1} \tilde{\boldsymbol{c}}^{\top} \boldsymbol{s}_{1} \boldsymbol{s}_{1}^{\top} \tilde{\boldsymbol{c}}}{\tilde{\boldsymbol{c}}^{\top}\left(\tilde{\boldsymbol{A}}_{1}+\tilde{\sigma}^{2} \boldsymbol{I}\right) \tilde{\boldsymbol{c}}} .
\end{aligned}
$$

Inserting $\tilde{\boldsymbol{c}}=\beta \tilde{\boldsymbol{A}}_{1}^{-1} \boldsymbol{s}_{1}$ yields

$$
\mathrm{SIR}_{1}=\frac{p_{1}\left(\boldsymbol{s}_{1}^{\top} \tilde{\boldsymbol{A}}_{1}^{-1} \boldsymbol{s}_{1}\right)^{2}}{\boldsymbol{s}_{1}^{\top} \tilde{\boldsymbol{A}}_{1}^{-1} \boldsymbol{s}_{1}+\tilde{\sigma}^{2} \boldsymbol{s}_{1}^{\top} \tilde{\boldsymbol{A}}_{1}^{-2} \boldsymbol{s}_{1}} .
$$

If we denote the value of the SIR of the desired (first) user obtained when $\tilde{\sigma}^{2}$ is used as $\operatorname{SIR}_{1}\left(\tilde{\sigma}^{2}\right)$, then it is not difficult to show that $\operatorname{SIR}_{1}\left(\tilde{\sigma}^{2}\right)$ is a decreasing function of $\tilde{\sigma}^{2}$. When $\tilde{\sigma}^{2}=0$, the highest value of SIR is attained. This result is expected because, at $\tilde{\sigma}^{2}=0$, the detector $\tilde{\boldsymbol{c}}$ is the MMSE multiuser detector and MMSE is known to maximize the SIR among all linear detectors [5]. When $\tilde{\sigma}^{2}$ is increased to $\sigma^{2}$, the decorrelating detector is reached.

\section{B. Estimating $\sigma^{2}$ at Every Filter Update}

In this section, we will investigate the convergence of the stochastic decorrelating detector algorithm where a new random unbiased estimate of $\sigma^{2}$ is used in every update of 


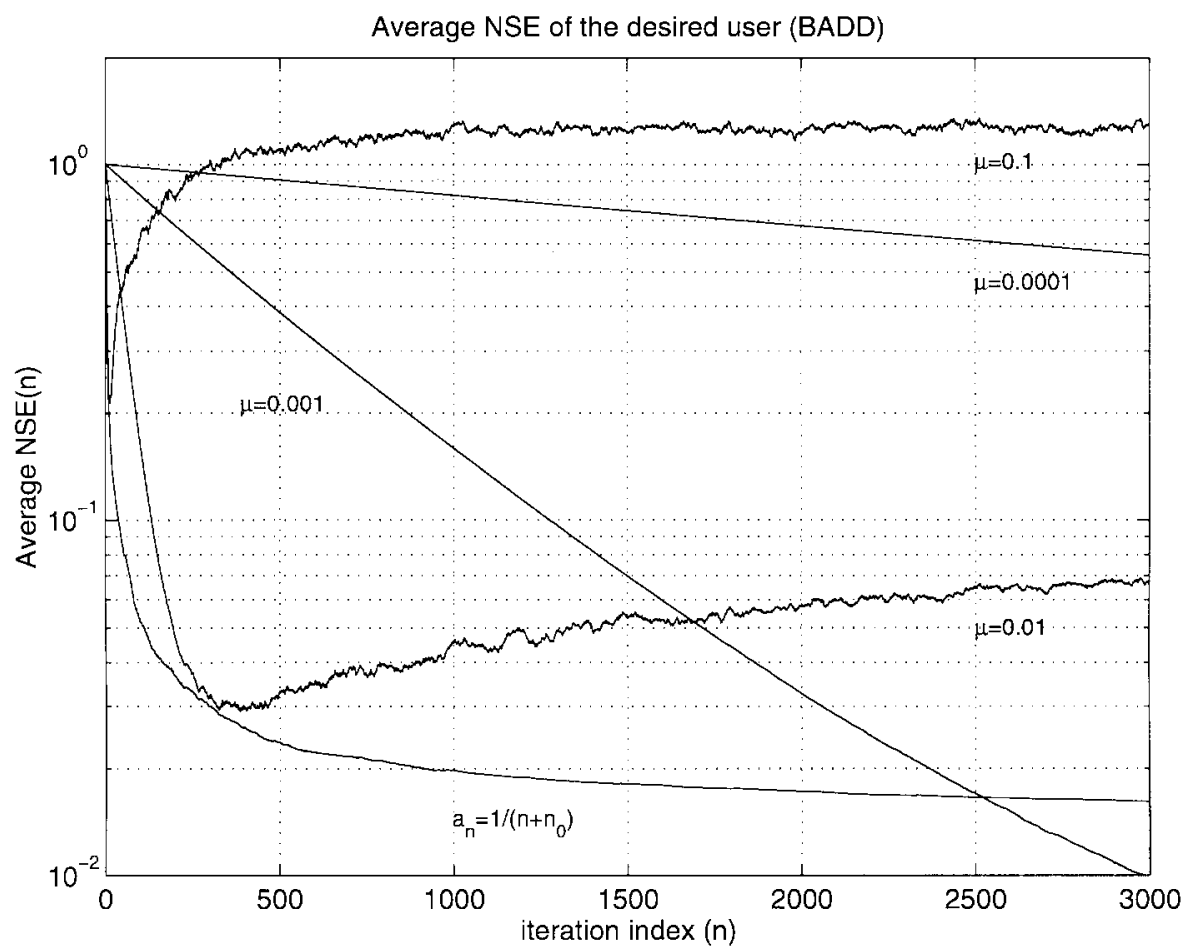

Fig. 2. Averaged normalized squared error (NSE) of the desired user.

the filter coefficients. Let $\hat{\sigma}^{2}(n)$ denote the estimate of $\sigma^{2}$ at iteration $n$, since we assume that the estimate is unbiased $E\left[\hat{\sigma}^{2}(n)\right]=\sigma^{2}$. As discussed earlier, one possible way of obtaining such an estimate is to allocate a filter $\boldsymbol{v}$ that is orthogonal to all signatures being used. Then the square of the output of the filter is an unbiased estimate for $\sigma^{2}$. In this section we will assume: $\hat{\sigma}^{2}=\left(\boldsymbol{v}^{\top} \boldsymbol{n}\right)^{2}$.

The filter update equation is given as

$$
\boldsymbol{c}(n+1)=c(n)-\mu\left[\left(\boldsymbol{r}(n) \boldsymbol{r}^{\top}(n)-\hat{\sigma}^{2}(n) \boldsymbol{I}\right) \boldsymbol{c}(n)-\boldsymbol{s}_{1}\right]
$$

The fixed point of this algorithm would be $\hat{\boldsymbol{c}}$, for which the expected value of the correction term on the right-hand side equals zero: $\boldsymbol{A} \hat{\boldsymbol{c}}=\boldsymbol{s}_{1}$, the decorrelating detector. From (56) we have

$$
\begin{aligned}
\boldsymbol{c}(n+1)=\boldsymbol{c}(n)-\mu[ & \left(\boldsymbol{r}(n) \boldsymbol{r}^{\top}(n)-\boldsymbol{A}\right. \\
& \left.\left.+A-\hat{\sigma}^{2}(n) \boldsymbol{I}\right) \boldsymbol{c}(n)-\boldsymbol{s}_{1}\right] \\
=\boldsymbol{c}(n)-\mu\left[\boldsymbol{A}(\boldsymbol{c}(n)-\hat{\boldsymbol{c}})+\left(\boldsymbol{r}(n) \boldsymbol{r}^{\top}(n)\right.\right. & \left.\left.-\boldsymbol{A}-\hat{\sigma}^{2} \boldsymbol{I}\right) \boldsymbol{c}(n)\right] \\
=\boldsymbol{c}(n)-\mu[ & A(\boldsymbol{c}(n)-\hat{\boldsymbol{c}})+\boldsymbol{\theta}(n)]
\end{aligned}
$$

where $\boldsymbol{\theta}(n)=\left(\boldsymbol{r}(n) \boldsymbol{r}^{\top}(n)-\boldsymbol{A}-\hat{\sigma}^{2} \boldsymbol{I}\right) \boldsymbol{c}(n)$. Subtracting $\hat{\boldsymbol{c}}$ from both sides of the final equation in (57) and defining $\hat{\epsilon}(n)=\boldsymbol{c}(n)-\hat{\boldsymbol{c}}$ yields

$$
\hat{\boldsymbol{\epsilon}}(n+1)=\hat{\boldsymbol{\epsilon}}(n)-\mu[\boldsymbol{A} \hat{\boldsymbol{\epsilon}}(n)+\boldsymbol{\theta}(n)] .
$$

Comparing (58) with (17), we see that Lemma 2 applied to $\hat{\epsilon}(n)$ is still valid. In order to complete the convergence proof we need the following lemma, similar to Lemma 3.
Lemma 5: There exist $0<c_{0} \leq c_{1}<\infty$, such that

$$
0 \leq E\left[\|\boldsymbol{\theta}(n)\|^{2} \mid \hat{\boldsymbol{\epsilon}}(n)=\boldsymbol{\epsilon}\right] \leq c_{0}+c_{1}\|\boldsymbol{\epsilon}\|^{2} .
$$

The proof of Lemma 5 can be found in the Appendix.

Thus, if an unbiased estimate for the noise variance is available, the proposed algorithm that uses the random noise variance estimates in every filter update converges to the decorrelating detector in the MSE sense.

\section{Simulation Results}

In the simulations, we consider a synchronous CDMA system with $N=6$ users, using randomly generated signature sequences with a processing gain of $G=31$. Powers of the users are assigned so that the final SIR of all users will be $20 \mathrm{~dB}$. Thus, the power of the $i$ th user is found by $p_{i}=100 \sigma^{2}\left[\boldsymbol{\Gamma}^{-1}\right]_{i i}$ where $\boldsymbol{\Gamma}=\boldsymbol{S} \boldsymbol{S}^{\top}$ is the crosscorrelation matrix. The algorithm is run for 100 times and averaged results are plotted in the figures. In Fig. 2, we plot the averaged normalized squared error (NSE) of the filter coefficients of the desired (first) user versus iteration index $n$, where NSE at iteration $n$ is defined as

$$
\operatorname{NSE}(n)=\frac{\left\|c(n)-c^{*}\right\|^{2}}{\left\|c^{*}\right\|^{2}} .
$$

The curves in Fig. 2 correspond to the blind adaptive decorrelating detector algorithms with fixed step size for different step size values and that with a time dependent step size sequence of the form of $a_{n}=1 /\left(n+n_{0}\right)$ with $n_{0}=5$. We observe that, if the step size $\mu$ is too large, then the algorithm does not converge: see increasing NSE curve for $\mu=0.1$ in Fig. 2. We also observe that for large step size values the convergence 


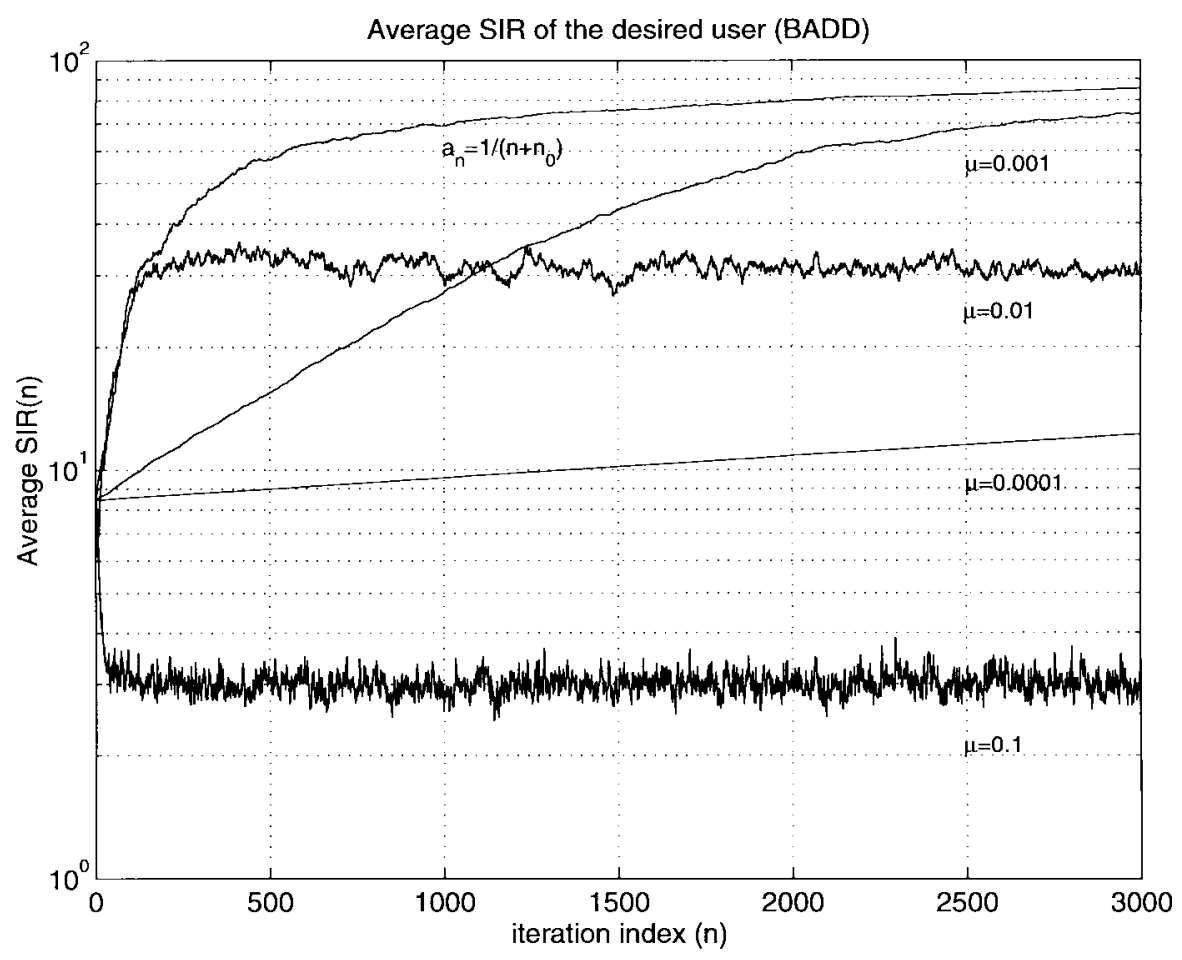

Fig. 3. Averaged SIR of the desired user.

rate is fast, but the limiting NSE is large too: compare the curves corresponding to $\mu=0.01$ and $\mu=0.001$ in Fig. 2.

Averaged (over 100 runs) SIR of the desired (first) user is plotted in Fig. 3 for the same system. At iteration $n$, the SIR of the desired user is calculated as

$$
\operatorname{SIR}(n)=\frac{p_{1}\left(\boldsymbol{s}_{1}^{\top} \boldsymbol{c}(n)\right)^{2}}{\sum_{j \neq 1} p_{j}\left(\boldsymbol{s}_{j}^{\top} \boldsymbol{c}(n)\right)^{2}+\sigma^{2}\left(\boldsymbol{c}^{\top}(n) \boldsymbol{c}(n)\right)}
$$

We observe that the convergence of the SIR's to the target SIR (which is $20 \mathrm{~dB}$ in this experiment) is quantitatively similar to the convergence of the NSE to zero.

The blind adaptive decorrelating detector of [10] is also implemented for the same system, and the SIR of the desired user is plotted as a function of iteration index in Fig. 4 for different values of the forgetting factor, $\beta$ (see [14]). In comparing Figs. 3 and 4, we see that for this instance the blind adaptive decorrelating detector in this work outperforms that of [10].

The aim of the following experiment is to verify the theoretical results of Section VI by running the proposed algorithm with a mismatch in the noise variance. First, we use the approach detailed in Section VI-A, where we obtain an estimate for $\sigma^{2}$ once before the bit transmission starts and use this estimate in every filter update as in (45). The algorithm is run for three values of $\tilde{\sigma}^{2}: \tilde{\sigma}^{2}=\beta \sigma^{2}$ for $\beta=1$ (exact knowledge on $\sigma^{2}$ ), $\beta=0.8$ (20\% error in estimation), and $\beta=0.5$ (50\% error in estimation). Fig. 5 shows the average SIR in these three cases, along with the convergence point SIR, calculated from (55) as horizontal lines. In order to make the difference where SIR's converge visible, we assumed a lower target SIR of $5 \mathrm{~dB}(\approx 3.16)$ and a highly loaded system $(N=20)$ for the same processing gain $(G=31)$. We observe from Fig. 5, that the average SIR of the desired user converges to the value predicted by the theoretical result in (55).

We also ran the algorithm given in (56), where a new estimate of the noise variance is obtained at every update of the filter coefficients. We observed that the average SIR converges to the same value when the algorithm is run with perfect knowledge of the noise variance [algorithm in (13)]. The averaged SIR's in both cases follow almost exact trajectories. Since there is no visible difference between the SIR's in both cases, we do not include the figure in this paper.

\section{CONCLUSION}

In this paper we presented an iterative and distributed blind adaptive decorrelating detector algorithm, which is based on the observation of the available statistics, and studied its convergence. The update equation of the algorithm needs the observation of the chip sampled input signal before the receiver filter and the output of the filter with the current filter coefficients. For the implementation of the algorithm to construct the decorrelating receiver filter of a user, only two parameters are needed to be known: the user's signature sequence and the variance of the AWG receiver noise.

We studied the convergence of the proposed algorithm both for a fixed step size sequence and for a time-varying step size sequence. For the first case, we developed the conditions of having lower and upper bounds on the MSE and showed that as the step size goes to zero the algorithm converges in the MSE. For the second case, we directly proved the convergence in the MSE. 


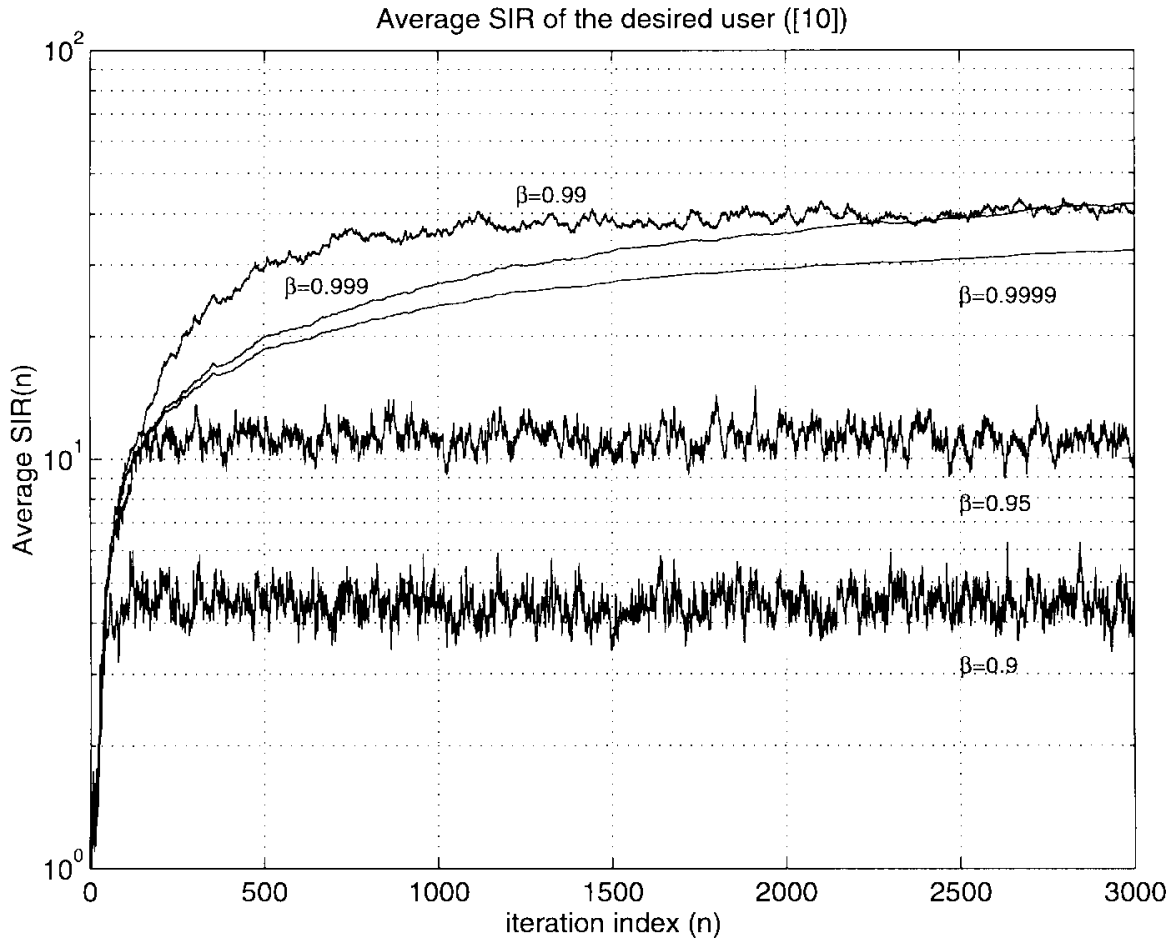

Fig. 4. Averaged SIR of the desired user (algorithm of [10]).

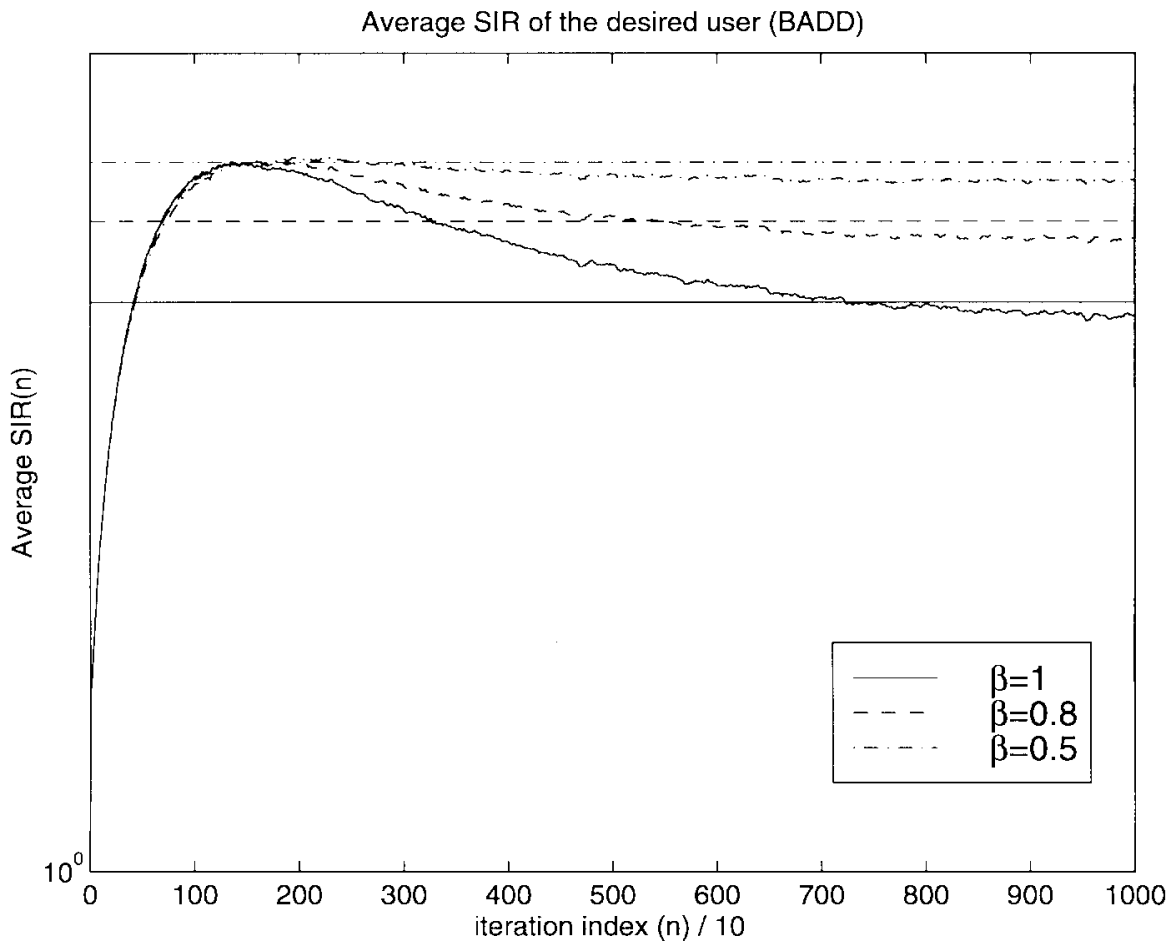

Fig. 5. Averaged SIR of the desired user with mismatch in noise variance. Noise variance estimate is calculated only once before the filter updates Noise variance used in the updates: $\tilde{\sigma}^{2}=\beta \sigma^{2}$.

\section{APPENDIX A:}

\section{ADDITIONAL PROOFS}

Proof of Lemma 1: We need to show that if $\boldsymbol{s}_{1}, \cdots, \boldsymbol{s}_{N}$ are linearly independent, then 1) any solution of (2) is also a solution of (6), and 2) any solution of (6) is also a solution of (2). Let $\hat{\boldsymbol{c}}$ be a solution of (2). Then $\boldsymbol{S} \hat{\boldsymbol{c}}=\alpha \boldsymbol{e}_{1}$. Multiplying both sides by first $\boldsymbol{A}$ and then with $\boldsymbol{S}^{\top}$ yields $\boldsymbol{S}^{\top} \boldsymbol{A} \boldsymbol{S} \hat{\boldsymbol{c}}=\alpha p_{1} \boldsymbol{s}_{1}$, and the proof of the first part is complete. Now let $\check{c}$ be a solution of (6). Then $\boldsymbol{S}^{\top} \boldsymbol{A} \boldsymbol{S} \breve{c}=\alpha p_{1} \boldsymbol{s}_{1}$ and equivalently

$$
\boldsymbol{S}^{\top}\left(\boldsymbol{A} \check{\boldsymbol{c}}-\alpha p_{1} \boldsymbol{e}_{1}\right)=\mathbf{0} .
$$

Note that any vector multiplying $S^{\top}$ yields a linear super- 
position of the columns of $\boldsymbol{S}^{\top}$, i.e., $\boldsymbol{S}^{\top} \boldsymbol{v}=\sum_{i=1}^{N} a_{i} \boldsymbol{s}_{i}$, for any vector $\boldsymbol{a}$. Combined with the independence of $\boldsymbol{s}_{1}, \cdots, \boldsymbol{s}_{N}$, (62) implies

$$
\Lambda S \check{c}-\alpha p_{1} e_{1}=\mathbf{0} .
$$

Multiplying both sides of (63) with $\Lambda^{-1}$ yields $S \check{\boldsymbol{c}}=\alpha \boldsymbol{e}_{1}$, completing the second part of the proof.

Proof of Lemma 2: First note that $A$ is a positive semidefinite matrix and its eigenvalues are nonnegative. To be specific, since the rank of $A$ is $N$ which is typically much less than $G,(G-N)$ eigenvalues of $\boldsymbol{A}$ are zero and the remaining $N$ eigenvalues are positive. Since $A \boldsymbol{x} \in \mathcal{L}$ by (8), eigenvectors of $\boldsymbol{A}$ are either completely in $\mathcal{L}$ with positive eigenvalues, or completely out of $\mathcal{L}$ (meaning that their projections onto $\mathcal{L}$ are zero) with zero eigenvalues. In this case, Lemma 2 is a simple result of Rayleigh quotient [15], which states that for any symmetric matrix $\boldsymbol{M}$ and vector $\boldsymbol{x}$

$$
\lambda_{\text {min }}^{M}\|\boldsymbol{x}\|^{2} \leq \boldsymbol{x}^{\top} \boldsymbol{M} \boldsymbol{x} \leq \lambda_{\max }^{M}\|\boldsymbol{x}\|^{2}
$$

where $\lambda_{\min }^{M}$ and $\lambda_{\max }^{M}$ are the smallest and the largest eigenvalues of $\boldsymbol{M}$. Note that in (20) $k_{1}$ and $k_{0}$ are equal to the largest and the smallest nonzero eigenvalues of $A$.

Proof of Lemma 3: Using (15), and noting that $\boldsymbol{\epsilon}(n)=$ $c(n)-\boldsymbol{c}^{*}$, we observe that

$$
\begin{aligned}
E\left[\|\boldsymbol{\eta}(n)\|^{2} \mid \boldsymbol{\epsilon}(n)=\boldsymbol{\epsilon}\right]=\left(\boldsymbol{\epsilon}+\boldsymbol{c}^{*}\right)^{\top} E[ & \left(\boldsymbol{r r}^{\top}-\left(\boldsymbol{A}+\sigma^{2} \boldsymbol{I}\right)\right)^{\top} \\
& \left.\cdot\left(\boldsymbol{r} \boldsymbol{r}^{\top}-\left(A+\sigma^{2} \boldsymbol{I}\right)\right)\right] \\
& \cdot\left(\boldsymbol{\epsilon}+\boldsymbol{c}^{*}\right) .
\end{aligned}
$$

Note that $E\left[\boldsymbol{r r ^ { \top }}\right]=\boldsymbol{A}+\sigma^{2} \boldsymbol{I}$ from (11). Applying this result to $(65)$ yields

$$
\begin{aligned}
E\left[\|\boldsymbol{\eta}(n)\|^{2} \mid \boldsymbol{\epsilon}(n)=\boldsymbol{\epsilon}\right]=\left(\boldsymbol{\epsilon}+\boldsymbol{c}^{*}\right)^{\top}\{ & E\left[\boldsymbol{r r}^{\top} \boldsymbol{r} \boldsymbol{r}^{\top}\right] \\
& \left.-\left(\boldsymbol{A}+\sigma^{2} \boldsymbol{I}\right)^{2}\right\} \\
& \cdot\left(\boldsymbol{\epsilon}+\boldsymbol{c}^{*}\right) .
\end{aligned}
$$

First, we will evaluate $E\left[\boldsymbol{r} \boldsymbol{r}^{\top} \boldsymbol{r} \boldsymbol{r}^{\top}\right]$ below. Using (10), we note that

$$
\begin{aligned}
\boldsymbol{r r}^{\top}= & \sum_{k} \sum_{l} \sqrt{p_{k}} \sqrt{p_{l}} a_{k} a_{l} \boldsymbol{s}_{k} \boldsymbol{s}_{l}^{\top}+\left(\sum_{k} \sqrt{p_{k}} a_{k} \boldsymbol{s}_{k}\right) \boldsymbol{n}^{\top} \\
& +\boldsymbol{n}\left(\sum_{k} \sqrt{p_{k}} a_{k} \boldsymbol{s}_{k}^{\top}\right)+\boldsymbol{n} \boldsymbol{n}^{\top}
\end{aligned}
$$

Therefore

$$
\begin{aligned}
\boldsymbol{r} \boldsymbol{r}^{\top} \boldsymbol{r} \boldsymbol{r}^{\top}= & \sum_{i} \sum_{j} \sum_{k} \sum_{l} \sqrt{p_{i}} \sqrt{p_{j}} \sqrt{p_{k}} \sqrt{p_{l}} a_{i} a_{j} a_{k} \\
& \cdot a_{l} \boldsymbol{s}_{i} \boldsymbol{s}_{j}^{\top} \boldsymbol{s}_{k} \boldsymbol{s}_{l}^{\top} \\
& +\sum_{i} \sum_{j} \sum_{k} \sqrt{p_{i}} \sqrt{p_{j}} \sqrt{p_{k}} a_{i} a_{j} a_{k} \boldsymbol{s}_{i} \boldsymbol{s}_{j}^{\top} \boldsymbol{s}_{k} \boldsymbol{n}^{\top} \\
& +\sum_{i} \sum_{j} \sum_{k} \sqrt{p_{i}} \sqrt{p_{j}} \sqrt{p_{k}} a_{i} a_{j} a_{k} \boldsymbol{s}_{i} \boldsymbol{s}_{j}^{\top} \boldsymbol{n} \boldsymbol{s}_{k}^{\top} \\
& +\sum_{i} \sum_{j} \sqrt{p_{i}} \sqrt{p_{j}} a_{i} a_{j} \boldsymbol{s}_{i} \boldsymbol{s}_{j}^{\top} \boldsymbol{n} \boldsymbol{n}^{\top}
\end{aligned}
$$

$$
\begin{aligned}
& +\sum_{i} \sum_{j} \sum_{k} \sqrt{p_{i}} \sqrt{p_{j}} \sqrt{p_{k}} a_{i} a_{j} a_{k} \boldsymbol{s}_{i} \boldsymbol{n}^{\top} \boldsymbol{s}_{j} \boldsymbol{s}_{k}^{\top} \\
& +\sum_{i} \sum_{j} \sqrt{p_{i}} \sqrt{p_{j}} a_{i} a_{j} \boldsymbol{s}_{i} \boldsymbol{n}^{\top} \boldsymbol{s}_{j} \boldsymbol{n}^{\top} \\
& +\sum_{i} \sum_{j} \sqrt{p_{i}} \sqrt{p_{j}} a_{i} a_{j} \boldsymbol{s}_{i} \boldsymbol{n}^{\top} \boldsymbol{n} \boldsymbol{s}_{j} \\
& +\sum_{i} \sqrt{p_{i}} a_{i} \boldsymbol{s}_{i} \boldsymbol{n}^{\top} \boldsymbol{n} \boldsymbol{n}^{\top} \\
& +\sum_{i} \sum_{j} \sum_{k} \sqrt{p_{i}} \sqrt{p_{j}} \sqrt{p_{k}} a_{i} a_{j} a_{k} \boldsymbol{n} \boldsymbol{s}_{i}^{\top} \boldsymbol{s}_{j} \boldsymbol{s}_{k}^{\top} \\
& +\sum_{i} \sum_{j} \sqrt{p_{i}} \sqrt{p_{j}} a_{i} a_{j} \boldsymbol{n} \boldsymbol{s}_{i}^{\top} \boldsymbol{s}_{j} \boldsymbol{n}^{\top} \\
& +\sum_{i} \sum_{j} \sqrt{p_{i}} \sqrt{p_{j}} a_{i} a_{j} \boldsymbol{n} \boldsymbol{s}_{i}^{\top} \boldsymbol{n} \boldsymbol{s}_{j}^{\top} \\
& +\sum_{i} \sqrt{p_{i}} a_{i} \boldsymbol{n} \boldsymbol{s}_{i}^{\top} \boldsymbol{n} \boldsymbol{n}^{\top} \\
& +\boldsymbol{n} \boldsymbol{n}^{\top} \sum_{i} \sum_{j} \sqrt{p_{i}} \sqrt{p_{j}} a_{i} a_{j} \boldsymbol{s}_{i} \boldsymbol{s}_{j}^{\top} \\
& +\boldsymbol{n} \boldsymbol{n}^{\top} \sum_{i} \sqrt{p_{i}} a_{i} \boldsymbol{s}_{i} \boldsymbol{n}^{\top}+\boldsymbol{n} \boldsymbol{n}^{\top} \boldsymbol{n} \sum_{i} \sqrt{p_{i}} a_{i} \boldsymbol{s}_{i}^{\top} \\
& +\boldsymbol{n} \boldsymbol{n}^{\top} \boldsymbol{n} \boldsymbol{n}^{\top} .
\end{aligned}
$$

Let $T_{i}$ denote the $i$ th term on the right-hand side of (68). Note that

$$
\begin{aligned}
E\left[T_{i}\right] & =0 \quad i=2,3,5,8,9,12,14,15 \\
E\left[T_{i}\right] & =\sigma^{2} \boldsymbol{A} \quad i=4,6,11,13 \\
E\left[T_{7}\right] & =\sigma^{2} G \boldsymbol{A} \\
E\left[T_{10}\right] & =\sigma^{2} p_{T} \boldsymbol{I} \\
E\left[T_{16}\right] & =(G+1) \sigma^{4} \boldsymbol{I} \\
E\left[T_{1}\right] & =A^{2}+\boldsymbol{C}
\end{aligned}
$$

where $p_{T}>0$ is the total receiver power, i.e., $p_{T}=\Sigma_{i} p_{i}$, and $C$ is given as

$$
\boldsymbol{C}=\sum_{i} \sum_{j \neq i} p_{i} p_{j} \boldsymbol{s}_{i} \boldsymbol{s}_{i}^{\top}+\sum_{i} \sum_{j \neq i} p_{i} p_{j} \boldsymbol{s}_{i} \boldsymbol{s}_{i}^{\top} \boldsymbol{s}_{j} \boldsymbol{s}_{j}^{\top}
$$

Note that since

$$
C=\frac{1}{2} \sum_{i} \sum_{j \neq i} p_{i} p_{j}\left(\boldsymbol{s}_{i} \boldsymbol{s}_{i}^{\top}+\boldsymbol{s}_{j} \boldsymbol{s}_{j}^{\top}\right)^{2}
$$

$C$ is positive semidefinite and symmetric. Combining the results in (69) yields

$E\left[\boldsymbol{r r}^{\top} \boldsymbol{r} \boldsymbol{r}^{\top}\right]=\boldsymbol{A}^{2}+\boldsymbol{C}+(G+4) \sigma^{2} \boldsymbol{A}+\sigma^{2} p_{T} \boldsymbol{I}+(G+1) \sigma^{4} \boldsymbol{I}$.

Inserting (72) into (66) and defining

$$
\boldsymbol{B}=\boldsymbol{C}+(G+2) \sigma^{2} \boldsymbol{A}+\sigma^{2} p_{T} \boldsymbol{I}+G \sigma^{4} \boldsymbol{I}
$$

yields

$$
E\left[\|\boldsymbol{\eta}(n)\|^{2} \mid \boldsymbol{\epsilon}(n)=\boldsymbol{\epsilon}\right]=\left(\boldsymbol{\epsilon}+\boldsymbol{c}^{*}\right)^{\top} \boldsymbol{B}\left(\boldsymbol{\epsilon}+\boldsymbol{c}^{*}\right) .
$$

Note that $B$ is a positive definite matrix, since $A$ and $C$ are positive semidefinite and $\sigma>0$. Using Rayleigh quotient [15], 
and denoting the smallest and largest eigenvalues of $\boldsymbol{B}$ by $\lambda_{\min }$ and $\lambda_{\max }$, we obtain

$$
\begin{aligned}
\lambda_{\min }\left\|\boldsymbol{\epsilon}+\boldsymbol{c}^{*}\right\|^{2} & \leq E\left[\|\boldsymbol{\eta}(n)\|^{2} \mid \boldsymbol{\epsilon}(n)=\boldsymbol{\epsilon}\right] \\
& \leq \lambda_{\max }\left\|\boldsymbol{\epsilon}+\boldsymbol{c}^{*}\right\|^{2} .
\end{aligned}
$$

Using the fact that $\|\boldsymbol{a}+\boldsymbol{b}\|^{2} \leq 2\left(\|\boldsymbol{a}\|^{2}+\|\boldsymbol{b}\|^{2}\right)$, for any two vectors $\boldsymbol{a}$ and $\boldsymbol{b}$, we obtain the desired result

$$
0 \leq E\left[\|\eta(n)\|^{2} \mid \boldsymbol{\epsilon}(n)=\boldsymbol{\epsilon}\right] \leq c_{0}+c_{1}\|\epsilon\|^{2}
$$

where $c_{0}=2 \lambda_{\max }\left\|c^{*}\right\|^{2}$ and $c_{1}=2 \lambda_{\max }$.

Proof of Lemma 4: We need only the upper bound given in (30). Replacing $\mu$ with $a_{n}$ we get

$$
b_{n+1} \leq\left(1-2 a_{n} k_{0}+\left(k_{1}^{2}+c_{1}\right) a_{n}^{2}\right) b_{n}+c_{0} a_{n}^{2} .
$$

We will follow Sakrison's approach [16, pp. 60-61] in the following derivation. Since $a_{n}$ is a monotonically decreasing sequence, there exists $n_{0}$ and $\delta, 0<\delta<2$, such that for $n \geq n_{0}$

$$
\left(1-2 k_{0} a_{n}+\left(k_{1}^{2}+c_{1}\right) a_{n}^{2}\right) \leq\left(1-(2-\delta) k_{0} a_{n}\right) .
$$

Furthermore, we can choose $n_{0}$ such that for $n \geq n_{0}$ we have $\left(1-(2-\delta) k_{0} a_{n}\right)>0$. For $n \geq n_{0}$, the inequality (77) can be further bounded as

$$
b_{n+1} \leq\left(1-(2-\delta) k_{0} a_{n}\right) b_{n}+a_{n}^{2} c_{0} .
$$

Starting at $n=n_{0}$ and executing the recursion repeatedly yields

$$
b_{n} \leq b_{n_{0}} \beta_{n_{0}, n-1}+c_{0} \sum_{k=n_{0}}^{n-1} a_{k}^{2} \beta_{k+1, n-1}
$$

where

$$
\beta_{k, n}= \begin{cases}\prod_{j=k}^{n}\left[1-a_{j}(2-\delta) k_{0}\right], & 0 \leq k \leq n \\ 1, & k>n .\end{cases}
$$

For $0<x<1$, we can use the inequality $\ln (1-x) \leq-x$ to show

$$
\begin{aligned}
\beta_{k, n} & =\exp \left(\sum_{j=k}^{n} \ln \left[1-a_{j}(2-\delta) k_{0}\right]\right) \\
& \leq \exp \left\{-(2-\delta) k_{0} \sum_{j=k}^{n} a_{j}\right\} .
\end{aligned}
$$

By the first condition in (38), and the fact that $(2-\delta) k_{0}>0$, the exponent in the above equation diverges to negative infinity and we have

$$
\lim _{n \rightarrow \infty} \beta_{k, n-1}=0 .
$$

This implies that $b_{n_{0}} \beta_{n_{0}, n-1}$ on the right-hand side of (80) goes to zero as $n$ goes to infinity. Now we will investigate the second term. Let $u(x)$ be the unit step function whose value is 1 for nonnegative $x$ and 0 otherwise. Then

$$
\begin{aligned}
\lim _{n \rightarrow \infty} & c_{0} \sum_{k=n_{0}}^{n-1} a_{k}^{2} \beta_{k+1, n-1} \\
= & c_{0} \lim _{n \rightarrow \infty} \sum_{k=n_{0}}^{\infty} a_{k}^{2} \beta_{k+1, n-1} u(n-1-k) \\
= & c_{0} \sum_{k=n_{0}}^{\infty} a_{k}^{2} \lim _{n \rightarrow \infty} \beta_{k+1, n-1} u(n-1-k) \\
= & 0
\end{aligned}
$$

since $\lim _{n \rightarrow \infty} \quad \beta_{k, n-1}=0$ from (83). We could exchange the limit and summation to obtain (85) from (84), because the sum on the right side of (84) is absolutely convergent. Finally, combining the results in (83) and (86) and the fact that $b_{n}$ is a nonnegative sequence, we obtain $\lim _{n \rightarrow \infty} b_{n}=0$ which concludes the proof.

Proof of Lemma 5: Note that $\boldsymbol{\theta}(n)$ can be written as

$$
\begin{aligned}
\boldsymbol{\theta}(n) & =\left(\boldsymbol{r}(n) \boldsymbol{r}^{\top}(n)-\boldsymbol{A}-\hat{\sigma}^{2} \boldsymbol{I}\right) \boldsymbol{c}(n) \\
& =\left(\boldsymbol{r}(n) \boldsymbol{r}^{\top}(n)-\left(\boldsymbol{A}+\sigma^{2} \boldsymbol{I}\right)-\left(\hat{\sigma}^{2}-\sigma^{2}\right) \boldsymbol{I}\right) \boldsymbol{c}(n) \\
& =\left(\boldsymbol{r}(n) \boldsymbol{r}^{\top}(n)-\left(\boldsymbol{A}+\sigma^{2} \boldsymbol{I}\right)-x \boldsymbol{I}\right) \boldsymbol{c}(n)
\end{aligned}
$$

where $x=\hat{\sigma}^{2}-\sigma^{2}$ is a zero mean random variable. Note that $x$ is given as $x=\left(\boldsymbol{v}^{\top} \boldsymbol{n}\right)^{2}-\sigma^{2}$ where $\boldsymbol{v}^{\top} \boldsymbol{s}_{i}=0$ for all $i$. By steps similar to those in the proof of Lemma 3, we get

$$
\begin{aligned}
E\left[\|\boldsymbol{\theta}(n)\|^{2} \mid \hat{\boldsymbol{\epsilon}}(n)=\boldsymbol{\epsilon}\right]= & (\boldsymbol{\epsilon}+\hat{\boldsymbol{c}})^{\top}\left\{E\left[\boldsymbol{r r}^{\top} \boldsymbol{r} \boldsymbol{r}^{\top}\right]-\left(A+\sigma^{2} \boldsymbol{I}\right)^{2}\right. \\
& \left.-2 E\left[x \boldsymbol{r} \boldsymbol{r}^{\top}\right]+E\left[x^{2}\right] \boldsymbol{I}\right\} \\
& \cdot(\boldsymbol{\epsilon}+\hat{\boldsymbol{c}}) .
\end{aligned}
$$

From the proof of Lemma 3, we have

$$
E\left[\boldsymbol{r} \boldsymbol{r}^{\top} \boldsymbol{r} \boldsymbol{r}^{\top}\right]-\left(\boldsymbol{A}+\sigma^{2} \boldsymbol{I}\right)^{2}=\boldsymbol{B}
$$

where $B$ is a positive definite matrix. We need to evaluate $E\left[x \boldsymbol{r} \boldsymbol{r}^{\top}\right]$ and $E\left[x^{2}\right]$. From (67), and the fact that $E[x]=0$, we can see that $E\left[x \boldsymbol{r} \boldsymbol{r}^{\top}\right]=E\left[x \boldsymbol{n} \boldsymbol{n}^{\top}\right]$. Then it is not difficult to show that

$$
E\left[x \boldsymbol{r} \boldsymbol{r}^{\top}\right]=E\left[\left\{\left(\boldsymbol{v}^{\top} \boldsymbol{n}\right)^{2}-\sigma^{2}\right\} \boldsymbol{n} \boldsymbol{n}^{\top}\right]=2 \sigma^{4} \boldsymbol{v} \boldsymbol{v}^{\top} .
$$

Similarly, it can be shown that

$$
E\left[x^{2}\right]=2 \sigma^{4} .
$$

Inserting (89)-(91) into (88) yields

$$
\begin{aligned}
& E\left[\|\boldsymbol{\theta}(n)\|^{2} \mid \hat{\boldsymbol{\epsilon}}(n)=\boldsymbol{\epsilon}\right] \\
& \quad=(\boldsymbol{\epsilon}+\hat{\boldsymbol{c}})^{\top}\left\{\boldsymbol{B}-4 \sigma^{4} \boldsymbol{v} \boldsymbol{v}^{\top}+2 \sigma^{4} \boldsymbol{I}\right\}(\boldsymbol{\epsilon}+\hat{\boldsymbol{c}}) .
\end{aligned}
$$

Note that $P \hat{\boldsymbol{\epsilon}}(n) \neq \mathbf{0}$, thus $\hat{\boldsymbol{\epsilon}} \in \mathcal{L}$ almost surely, and $(\boldsymbol{\epsilon}+\hat{\boldsymbol{c}})^{\top} \boldsymbol{v} \boldsymbol{v}^{\top}(\boldsymbol{\epsilon}+\hat{\boldsymbol{c}})=\left[(\boldsymbol{\epsilon}+\hat{\boldsymbol{c}})^{\top} \boldsymbol{v}\right]^{2}=0$ when $\boldsymbol{P} \hat{\boldsymbol{\epsilon}}(n) \neq \mathbf{0}$. Thus, (92) can be written as

$$
E\left[\|\boldsymbol{\theta}(n)\|^{2} \mid \hat{\boldsymbol{\epsilon}}(n)=\boldsymbol{\epsilon}\right]=(\boldsymbol{\epsilon}+\hat{\boldsymbol{c}})^{\top}\left\{\boldsymbol{B}+2 \sigma^{4} \boldsymbol{I}\right\}(\boldsymbol{\epsilon}+\hat{\boldsymbol{c}}) .
$$

Since $B$ is positive definite, the desired result follows. 


\section{REFERENCES}

[1] S. Verdú, "Multiuser detection," Adv. Statis. Signal Processing, vol. 2, pp. 369-409, 1993

[2] _ "Minimum probability of error for asynchronous Gaussian multiple-access channels," IEEE Trans. Inform. Theory, vol. 32, pp. 85-96, Jan. 1986.

[3] R. Lupas and S. Verdú, "Linear multiuser detectors for synchronous code-division multiple-access channels," IEEE Trans. Inform. Theory, vol. 35, pp. 123-136, Jan. 1989.

[4] A. Duel-Hallen, "Decorrelating decision-feedback multiuser detector for synchronous code-division multiple-access channels," IEEE Trans. Commun., vol. 41, pp. 285-290, Feb. 1993.

[5] U. Madhow and M. L. Honig. "MMSE interference suppression for direct-sequence spread-spectrum CDMA," IEEE Trans. Commun., vol. 42, pp. 3178-3188, Dec. 1994.

[6] M. K. Varanasi and B. Aazhang, "Multistage detection in asynchronous code-division multiple-access communications," IEEE Trans Commun., vol. 38, pp. 509-519, Apr. 1990.

[7] R. Lupas and S. Verdú. "Near-far resistance of multiuser detectors in asynchronous channels," IEEE Trans. Commun., vol. 38, pp. 496-508, Apr. 1990.

[8] M. Honig, U. Madhow, and S. Verdú, "Blind adaptive multiuser detection," IEEE Trans. Inform. Theory, vol. 41, pp. 944-960, July 1995.

[9] D. S. Chen and S. Roy, "An adaptive multiuser receiver for CDMA systems," IEEE J. Select. Areas Commun., vol. 12, pp. 808-816, Jun. 1994.

[10] X. Wang and H. V. Poor, "Blind adaptive interference suppression for CDMA communications based on eigenspace tracking," in Proc. Conf. Information Sciences and Systems, CISS'97, Mar. 1997, pp. 468-473.

[11] W. F. Stout, Almost Sure Convergence. New York: Academic, 1974.

[12] L. Gyorfi, "Adaptive linear procedures under general conditions," IEEE Trans. Inform. Theory, vol. IT-30, pp. 262-267, Mar. 1984.

[13] H. Robbins and S. Monro, "A stochastic approximation method," Ann. Math. Statis., vol. 22, pp. 400-407, 1951 .

[14] B. Yang, "Projection approximation subspace tracking," IEEE Trans. Signal Processing, vol. 43, pp. 95-107, Jan. 1995.
[15] G. Strang, Linear Algebra and Its Applications, 3rd ed. San Diego, CA: Saunders, 1988

[16] D. J. Sakrison, "Stochastic approximation: A recursive method for solving regression problems," in Advances in Communication Systems 2, A. V. Balakrishnan, Ed. New York: Academic, 1966, pp. 51-106.

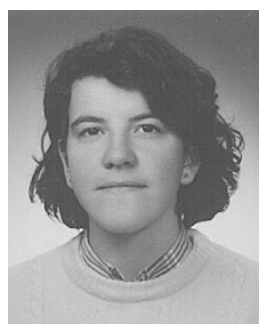

Sennur Ulukus (S'93) received the B.S. and M.S degrees in electrical and electronics engineering from Bilkent University, Ankara, Turkey, in 1991 and 1993, respectively, and the Ph.D. degree in electrical and computer engineering from Rutgers University, Piscataway, NJ, in 1998.

While studying for the Ph.D. degree she was with the Wireless Network Laboratory (WINLAB) at Rutgers University. Since September, 1998, she has been with the Wireless Communications Research Department at AT\&T Laboratories, Red Bank, NJ. Her research interests include power control, multiuser detection and array signal processing for wireless communication networks, and design and analysis of CDMA systems.

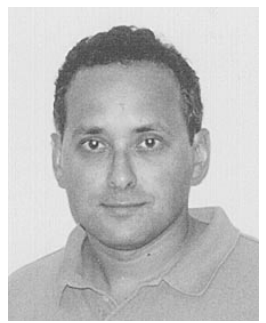

Roy D. Yates (M'91) received the B.S.E. degree in 1983 from Princeton University, Princeton, NJ, and the S.M. and Ph.D. degrees in electrical engineering from the Massachusetts Institute of Technology, Cambridge, in 1986 and 1990, respectively.

Since 1990, he has been with the Wireless Information Network Laboratory (WINLAB) in the Department of Electrical and Computer Engineering at Rutgers University, Piscataway, NJ, where he is currently an Associate Professor. His research interests include power control, handoff, multiaccess protocols, and multiuser detection for wireless networks. 